\title{
Probing the Helical Content of Growth Hormone-Releasing Factor Analogs Using Electrospray Ionization Mass Spectrometry
}

\author{
Cynthia L. Stevenson and Robert J. Anderegg \\ Glaxo Research Laboratories, Research Triangle Park, North Carolina, USA
}

\author{
Ronald T. Borchardt \\ Department of Pharmaceutical Chemistry, University of Kansas, Lawrence, Kansas, USA
}

\begin{abstract}
A series of growth hormone-releasing factor analogs have been studied by both circular dichroism and electrospray ionization mass spectrometry (ESI/MS). The peptides are 32 residues long and are known to adopt a random-coil structure in aqueous solution but become increasingly helical as the proportion of organic solvent is increased. Deuterium exchange was observed as an increase in mass of the peptide, as measured by ESI/MS. Rates of exchange were measured and half-lives calculated for analogs containing amino acid substitutions designed to promote or discourage helix formation. Exchange was slower in peptides that are helical (as shown by circular dichroism) than in randomly coiled peptides. Solution conditions that favor helix formation also produced slower exchange rates. These studies suggest that ESI/MS can provide date about the extent and stability of helix formation. (J Am Soc Mass Spectrom 1993, 4, 646-651)
\end{abstract}

$\mathrm{F}$ Tew developments in protein mass spectrometry have had as immediate and profound an effect on the field as has electrospray ionization mass spectrometry (ESL/MS) [1, 2]. The measurement of molecular mass up to approximately $100,000 \mathrm{Da}$ with very high precision is now routine. A number of recent reports [3-8] suggest that ESI/MS may provide information about protein secondary structure as well. Charged states are distributed differently in native proteins than in their denatured counterparts. Recently, Katta and Chait [5] demonstrated that ESI/MS could be used to measure deuterium exchange into proteins and might therefore yield another type of information about secondary structure. Although electrospray mass spectra may provide clues to secondary structure, it remains difficult to interpret that information. To simplify the probleir, we have been using ESI/MS to study a series of well-characterized peptide models.

One such system involves a series of growth hormone-releasing factor (GRF) analogs, which we have investigated in an effort to understand the relationship between primary sequence and secondary structure under a variety of solution conditions. GRF analogs have been widely studied and have been suggested as a therapy for growth hormone deficiency [9]. The native human GRF (hGRF) is a 44-residue hy-

Address reprint requests to Robert J. Anderegg, Glaxo Research Laboratories, 5 Moore Drive, Research 'Triangle Park, NC 27502. pothalamic peptide related to glucagon. An analog corresponding to residues $1-32$, with Leu in position 27 and a C-terminal amide ( $\mathrm{Leu}^{27}-\mathrm{hGRF}(1-32) \mathrm{NH}_{2}$ ), is shown in Scheme I. This peptide has been shown to adopt a random coil under aqueous conditions but becomes helical in solvent mixtures containing trifluoroethanol or methanol [10-14]. The structure has been described, using nuclear magnetic resonance (NMR) or modeling studies, as being composed of either one long helix [11] or two shorter helical regions [10], encompassing residues $6-13$ and $16-29$. The remainder of the peptide has been demonstrated to be a random coil; no other secondary structures have been observed. These data suggest that residues 13-16 may be in an unstable secondary structure and that the introduction of a helix-forming residue or a helixbreaking residue at position 15 might have a significant effect on overall helical structure.

$$
\begin{aligned}
& \text { Y-A-D-A-I-F-T-N-S-Y-R-K-V-L-G-Q-L- } \\
& \text { S-A-R-K-L-L-Q-D-I-L-S-R-Q-Q-G-NH }{ }_{2}
\end{aligned}
$$

Scheme I. Sequence of Leu ${ }^{27}-\mathrm{hGRF}(1-32) \mathrm{NH}_{2}$.

The $\alpha$-helix is the most abundant secondary structure type in proteins overall, although individual proteins may vary in their relative amounts of helix, $\beta$-sheet, or random coil. We believed that the GRF analogs would provide a well-characterized system with which to explore whether ESI/MS could be uti- 
lized to measure the extent of formation and/or the stability of helices in peptides. Helical content is generally determined using circular dichroism or NMR, but each of these techniques has its own shortcomings.

\section{Experimental}

\section{Materials}

GRF Analogs [ Leu $^{27}$-hGRF(1-32) $\mathrm{NH}_{2} ; \mathrm{Ala}^{15}, \mathrm{Leu}^{27}$ bovine GRF (bGRF)(1-32) NH ${ }_{2}$; and $\mathrm{Pro}^{15}, \mathrm{Leu}^{27_{-}}$ bCRF $(1-32) \mathrm{NH}_{2}$ ] were generous gifts of the Upjohn Company (Kalamazoo, MI). $\mathrm{D}_{2} \mathrm{O} \quad(99.996 \%)$ and deutero $\left(\mathrm{d}_{4}\right)$-methanol $(99.96 \%)$ were purchased from Cambridge Isotope Laboratories (Woburn, MA).

\section{Circular Dichroism}

Circular dichroism (CD) studies were performed on an AVIV 60DS spectrometer from Aviv Associates (Lakewood, NI). A 0.05-cm jacketed quartz cell from Hellma (Forest Hills, NY) was thermostated at $25^{\circ} \mathrm{C}$. Complete experimental details have been published elsewhere [15]. All spectra were collected on solutions with peptide concentrations ranging from 0.05 to 0.5 $\mathrm{mg} / \mathrm{mL}$, using a scan range of $190-250 \mathrm{~nm}$, a $0.25-\mathrm{nm}$ step size, and a bandwidth of $1.5 \mathrm{~nm}$. The mean residue ellipticity, $\theta$, at $222 \mathrm{~nm}$ was expressed in degrees $\mathrm{cm}^{2} \mathrm{dmol}^{-1}$. Percent helicity was calculated at $222 \mathrm{~nm}$, using a modified formula from Greenfield and Fasman [16]:

$$
\text { Fraction helix }=\frac{\theta_{\text {obs }}-\theta_{\text {coil }}}{\theta_{\text {helix }}-\theta_{\text {coil }}}
$$

where $\theta_{\text {coil }}=-2340$, and $\theta_{\text {helix }}=-30,300$ (values are from Chen and co-workers $[17,18]$ and Chakrabartty et al. [19], respectively).

\section{Mass Spectrometry}

Electrospray mass spectra were collected with a Sciex API-III mass spectrometer (Sciex, Thornhill, Ontario, Canada) in the positive ion mode. Samples were dissolved to a concentration of approximately $10 \mathrm{pmol} / \mu \mathrm{L}$ in methanol/water (or deuteromethanol/ $\mathrm{D}_{2} \mathrm{O}$ ) and transferred as quickly as possible ( $<1 \mathrm{~min}$ ) to a $100-\mu \mathrm{L}$ gastight syringe. Methanol concentrations were $0 \%$, $40 \%$, and $80 \%(\mathrm{v} / \mathrm{v})$. The solutions were unbuffered, and $\mathrm{pH}$ was not routinely measured; but the $\mathrm{pH}$ of an aqueous solution of the peptides before addition of methanol was 3.5. A Harvard model 22 syringe pump (Harvard Apparatus, South Natick, MA) was used to infuse the sample into the instrument at a rate of 2 $\mu \mathrm{L} / \mathrm{min}$. The ion spray needle was maintained at 4800 $\mathrm{V}$, and the orifice potential was $80 \mathrm{~V}$. A mass range of 900-970, encompassing the abundant +4 charged ion, was scanned continuously at $2.5 \mathrm{~s}$ per scan for a period of several minutes.

\section{Results and Discussion}

The model system chosen in which to study helicity was a pair of bGRF analogs and an hGRF analog, differing from each other only in positions 15 and 28 . The hGRT analog contained a Gly ${ }^{15}$ and a $\mathrm{Ser}^{28}$, whereas the bGRF analogs were substituted with either a Pro ${ }^{15}$ or an $\mathrm{Ala}^{15}$, but both contained an $\mathrm{Asn}^{28}$. It was expected that the $A 1^{15}{ }^{15}$-bGRF analug would show increased helicity [20] relative to the native sequence and that the Pro ${ }^{15}$ analog would show decreased helicity owing to the helix-breaking character of proline [20]. Gly and Pro residues are generally considered to be helix breaking, although the effect of Pro is more pronounced because Pro lacks an amide proton required to maintain the hydrogen bonding that provides the stabilization for the helix. The proline ring is also not easily accommodated into a helical structure and has been shown to distort the helix axis [21]. The effect on helical content of the second alteration in primary sequence, at position 28 , was considered minimal because of the apparent stability of the helical structure observed in two-dimensional NMR experiments [15]. Therefore, comparisons of helical content between the bGRF and hGRF analogs are probably justified.

The far-UV circular dichroic spectra of all GRF analogs studied showed characteristic $\alpha$-helical secondary structure (Figure 1). The appearance of the spectra at any given level of organic solvent was independent of concentration, ionic strength, and $\mathrm{pH}$ [15], indicating that the solvent-induced effect was far greater than the effects of $\mathrm{pH}$, concentration, or ionic strength. Felix et al. [22] have also shown by NMR that the helicity of GRF analogs in methanol was not affected by changes in $\mathrm{pH}$ over the range 3-6. Singlewavelength determinations at $222 \mathrm{~nm}$ were made and used to calculate $\theta_{222}$ and percent helicity. Methanol was chosen over 2,2,2-trifluoroethanol (TFE) because it

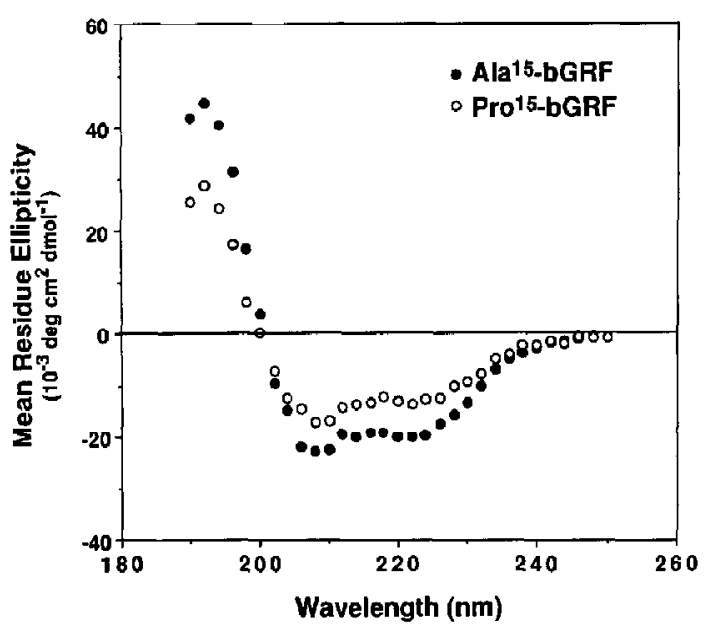

Figure 1. CD spectra of $\mathrm{Ala}^{15}$ - and Pro ${ }^{15}$-bGRF in $80 \%$ methanol. 
provided a weaker helix-inducing environment [23] in which single amino acid replacement analogs could be studied without being overshadowed by solventinduced effects. Although we did not specifically compare the results in TFE and methanol, NMR studies show that there is little difference between structure of GRF peptides in the two solvents [10-14].

Figure 1 shows the circular dichroic spectra for the $A l a^{15}$-and $\mathrm{Pro}^{15}$-bGRF analogs in $80 \%$ methanol. The $A l a^{15}$-bGRF analog showed greater helix-forming propensity ( $81 \%$ helix for $\mathrm{Ala}^{15}$-bGRF, $47 \%$ for Pro ${ }^{15}$. bGRF), as would be expected considering the greater helix-forming character of alanine compared with proline [20]. The $\mathrm{Ala}^{15}-\mathrm{bGRF}$ and $\mathrm{Gly}{ }^{15}$-hGRF analogs did not appear significantly different from each other, but both were considerably more helical than the Pro ${ }^{15}$ bGRF analog. This trend in helix-forming ability was observed throughout the range of methanol concentrations studied $(0-80 \%)$. These results suggest that residue 15 occupies a position easily modified to become helical, consistent with the findings of twodimensional NMR [14].

Other workers have shown that the GRF peptides adopt a random coil in aqueous solution but become more helical as the percentage of organic solvent increases [10-12]. As the percentage of water decreases, the hydrogen bonding to the solvent of peptide carbonyls and amide nitrogens decreases, leading to stronger intrachain hydrogen bonding and greater helical character [24]. This is consistent with our observations based on $\mathrm{CD}$. All three analogs showed a linear increase in percent helicity with increasing methanol concentration over the range $0-80 \%$ methanol. As the methanol concentration increased from $0 \%$ to $80 \%$, the percent helix of the Pro ${ }^{15}-b G R F$ analog increased from $7 \%$ to $47 \%$; that of the $\mathrm{Ala}^{15}$-bGRF analog changed from $18 \%$ to $81 \%$; that of the Gly ${ }^{15}$-hGRF analog changed from $12 \%$ to $77 \%$. Correlation coefficients for the three lines ranged from 0.980 to 0.997 .

The GRF analogs were then studied by ESI/MS $[1,2]$. This relatively recent and gentle ionization method, in which protein solutions are sprayed in the presence of a high electric field, leads to the formation of analyte ions, often bearing multiple charges (for a review of electrospray, see ref 1 ). Figure 2 shows the electrospray mass spectrum of the Pro ${ }^{15}-b G R F$ analog. The major ions are the +5 and +4 charged states at $m / z 744.9$ and 931.1, respectively. The charge on each ion and the mass of the intact peptide are determined in a straightforward fashion [25]. The accuracy and precision of molecular mass measurement for peptides of this size are quite good. In this example, a relative molecular mass of $3719.6 \pm 0.7$ was measured; the calculated relative molecular mass based on the amino acid sequence, is 3720.3 .

To study the helical content of the peptides, we used deuterium exchange in conjunction with ESI/MS [5]. Protons on peptides in a random coil will exchange rapidly with the surrounding solvent, whereas those

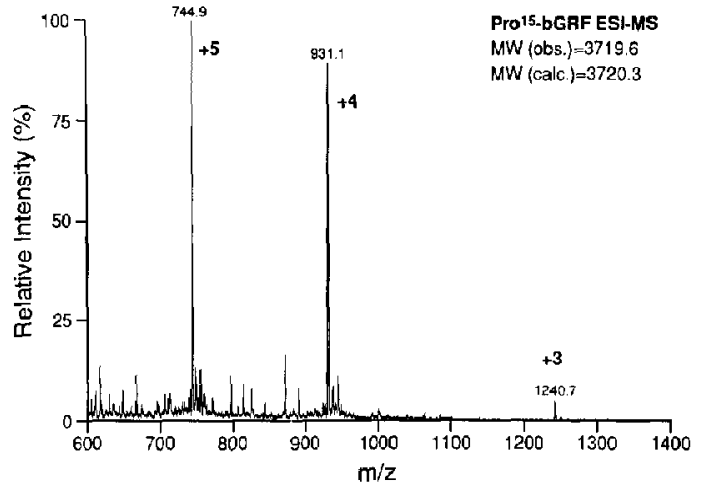

Figure 2. ESI mass spectrum of Pro ${ }^{15}$-bGRF.

involved in hydrogen bonds, such as the bonds that stabilize an $\alpha$-helix, will exchange more slowly. Deuterium exchange of proteins is often studied by NMR. In an ESI/MS experiment, the exchange of protons with deuterons will result in the increase in measured molecular mass of the peptide by 1 Da per exchange site. The GRF analogs have $68-70$ exchangeable protons and so, when fully exchanged, should show a shift in molecular mass of 68-70 Da.

It is important to note that although the ESI/MS detects ions in the gas phase, the exchange that is being measured is occurring in the liquid phase, before the gaseous ions are formed. Because of this, we believe that it is reasonable to compare the results of structural measurements from CD, NMR, and ESI/MS. Furthermore, with our current level of understanding, we cannot differentiate by ESI/MS hydrogen bonding in $\alpha$-helices from hydrogen bonding in $\beta$-sheets or other secondary structures. The GRF model was chosen because only one structural type (helix) is represented.

Katta and Chait [5] studied the exchange of bovine ubiquitin and observed a relatively slow exchange (62\% in $20 \mathrm{~min})$. The GRF analogs, presumably because of their small size, exchange much more quickly. Exchange proceeds rapidly during the first 3-4 min of the experiment and then reaches a plateau. To obtain information on a rapidly changing system, a short scan was desirable but one in which the maximum time was spent on the ions of interest. Consequently, experiments were set up to scan only a limited mass range ( $m / z$ 900-970), encompassing the abundant +4 charge state. Figure 3 shows the change in mass for the $\mathrm{Ala}^{15}$-bGRF analog as a function of time after exposure to deuterated solvent ( $80 \%$ methanol- $\mathrm{d}_{4}$ ). There is an almost instantaneous rise in mass as the protons in the amino acid side chains and at the extreme ends of the peptide backbone exchange. The mass increase then slows somewhat as another population of protons exchange; these most likely represent amide protons in the peptide backbone that are involved in hydrogen bonding. After approximately 3 min, the measured mass levels off or continues to 


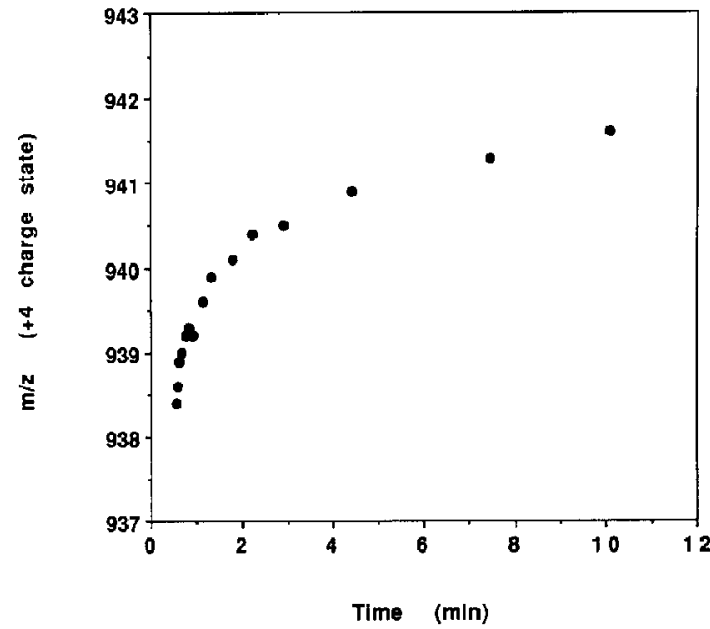

Figure 3. Change in mass-to-charge ratio of the +4 charged ion of $\mathrm{Ala}^{15}$-bGRF over time after exposure to deuterated solvent ( $80 \%$ methanol in water). The fully protonated ion would be $m / z$ 924.6, and the fully deuterated ion $m / z$ 943.0.

increase at a very slow rate. Experiments indicate that exchange of the last protons is still not complete even after several days. The very slowly exchanging protons probably represent amide backbone protons involved in very stable hydrogen bonds, as would be found deep within an $\alpha$-helix.

To obtain information about the rate of exchange, the data can be plotted as "percent hydrogen remaining" versus time (Figure 4). The exponential decay appears to represent pseudo-first-order kinetics and can be curve-fit to determine a rate constant and halflife. For the $\mathrm{Ala}^{15}$-bGRF analog data, the curve in Figure 4 shows the best fit to the expression

$$
y=A+B \times \exp (-C t)
$$

and predicts a half-life of $45 \mathrm{~s}$.

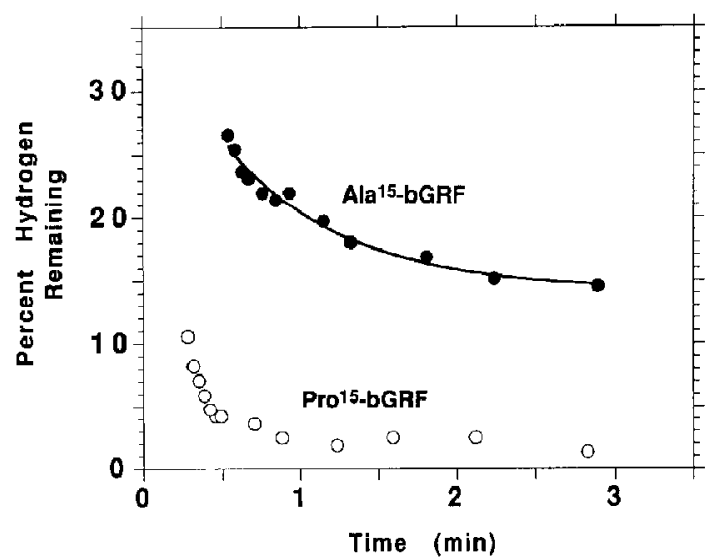

Figure 4. Deuterium exchange of $\mathrm{Pro}^{15}$ - and $\mathrm{Ala}^{15}-\mathrm{bGRF}$ in deuterated solvent ( $80 \%$ methanol in water). Curve, $y=14.2+$ $23.5 \exp (-1.3 \times t)$
Figure 4 compares the data for the exchange of the Pro $^{15}$ - and Ala ${ }^{15}$-bGRF analogs in $80 \%$ aqueous methanol (both solvents deuterated). The half-life for the Pro ${ }^{15}$-bGRF analog is very short $(\sim 5 \mathrm{~s})$ compared with $45 \mathrm{~s}$ for $\mathrm{Ala}^{15}$-bGRF. This is consistent with the slower exchange of the $A a^{15}-b G R F$ analog due to more extensive hydrogen bonding and, presumably, greater helical content. At lower methanol concentrations ( $40 \%$ or less), the exchange of the Pro ${ }^{15}$-bGRF analog was too fast to measure (i.e., at the first time point, the mass was that of the fully deuterated peptide). Once again, the rate of exchange of the Gly ${ }^{15}$-hGRF analog was similar to that of $\mathrm{Ala}^{15}$-bGRF, whereas both were slower than the Pro ${ }^{15}$-bGRF analog.

As with the CD studies, when the GRF analogs were studied by ESI/MS in varying concentrations of methanol, there was evidence of a marked change in structure. Figure 5 shows the exchange for the Ala ${ }^{15}$ bGRF analog in various solvent compositions. The exchange was slowest in $80 \%$ methanol and faster in $40 \%$ and $0 \%$ methanol, consistent with the peptide being most helical in the high proportion of organic solvent and becoming increasingly random as the organic content decreases. It should be noted that the rate of exchange is somewhat dependent on the solvent composition, irrespective of secondary structure, and the measured exchange rate is a composite of these two effects.

In an attempt to directly compare the results of the ESI/MS and CD experiments, one must first convert to some common unit of measure. CD data are usually reported as percent helicity; that is, the number of residues involved in the $\alpha$-helix divided by the total number of residues. The ESI/MS results are reported here as percent of hydrogens exchanged or remaining. If one assumes that all side-chain protons exchange very quickly and that the protons on the $\mathrm{N}$ - and C-termini of the peptide also exchange immediately, then one can calculate the number of amide protons likely to be involved in hydrogen bonding and, from that, the percent of the total amide protons in the helix.

For example, the $\mathrm{Ala}^{15}$-bGRF analog has a total of 70 exchangeable protons: 35 in side chains, 2 each at the $\mathrm{N}$ - and C-termini (the C-terminus is amidated), and 31 in the amide backbone. Only the latter are used to compute percent helicity. Of the amide backbone protons, the first four from the N-terminus cannot be involved in hydrogen bonding because they have no carbonyl partners and so would be expected to exchange immediately with solvent. In $80 \%$ methanol (deuterated), after $4 \mathrm{~min}$, we estimate that a total of 61 $(87 \%)$ hydrogens have exchanged (see Figure 4 ). Nine of a possible 31 amide protons are not exchanged and are therefore presumed to be involved in the helix. We also include the four protons at the start of the helix, which must be present, although we will not observe them in ESI/MS, bringing the total to 13 of a possible 32. (The N-terminus of the peptide was not included in the count of 31 amide protons but would be included 


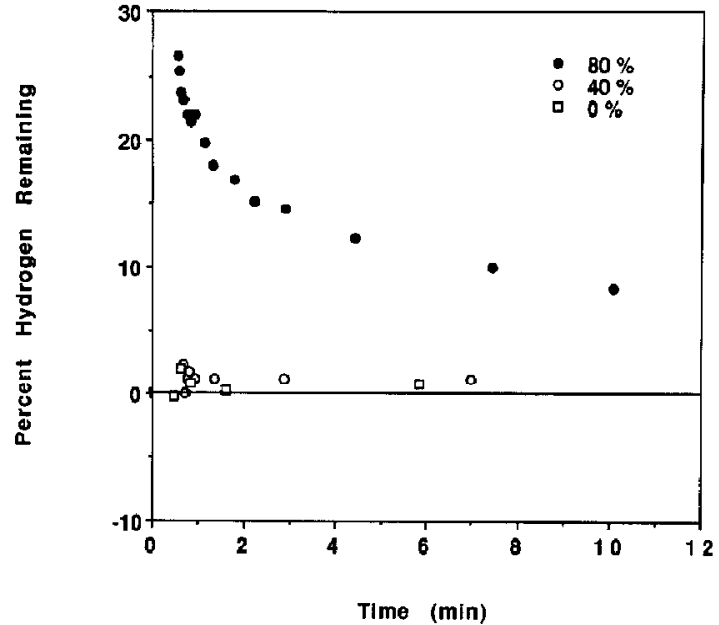

Figure 5. Deuterium exchange of $\mathrm{Ala}^{15}-\mathrm{bGRF}$ as a function of solvent composition. Data points taken at $0 \%, 40 \%$, and $80 \%$ methanol in water (all solvents deuterated).

in the total residues in the peptide.) We would report the helical content under these conditions as $41 \%$.

In this same concentration of methanol $(80 \%)$, the CD experiments measure a helical content of $81 \%$, significantly higher than the number obtained from ESI/MS. This pattern of underestimation by ESI/MS was observed for all analogs at all methanol concentrations and is therefore likely to be a real effect. We believe that the most reasonable explanation for this phenomenon is that $\mathrm{CD}$ measures a time-averaged helical content, whereas ESI/MS measures exchanged protons. If a portion of the helix was rapidly unfolding and refolding, CD would measure it as helical. With ESI/MS, however, as a portion of the helix unfolds, its protons would exchange, and, regardless of whether it refolded again or not, it would no longer be recorded as helical. It is likely that the magnitude of the discrepancy between the ESI/MS and CD results reflects the stability of the helix, although much more study will be required before such stability information is interpretable.

\section{Conclusions}

Hydrogen exchange followed by ESI/MS appears to be a sensitive and useful method for observing secondary structure in peptides. For the GRF analogs studied, the ESI/MS results were obtained with approximately $200 \mathrm{pmol}$ of peptide (700 ng per experiment) and correlated well with the data obtained by $C D$, although the estimates of percent helicity by ESI/MS are lower than those obtained by CD. Amino acid substitution that promotes (Ala) or inhibits (Pro) helix formation resulted in slower or faster exchange, respectively. Solution conditions known to promote helix formation caused slower exchange than observed for solutions in which the peptides are known to adopt a random configuration. ESI/MS measurements provide information about the rate of exchange and the extent of exchange. In combination with the information obtained from $C D$, the exchange data may yield clues as to both the amount and the stability of secondary structure. NMR measurements of the helicity of both sets of GRF analogs are under way and will be reported separately.

Both CD and ESI/MS measure global helicity rather than localizing where in the peptide the helix occurs. NMR can provide specific information about the involvement of each individual amino acid in the helix but requires more material than either CD or ESI/MS. In an effort to gain information about where the helical regions are, we are currently exploring tandem mass spectrometry (MS/MS) in conjunction with deuteriumexchange ESI/MS. The fact that the mass of the peptide appears to plateau after the first $5 \mathrm{~min}$ of the exchange experiment may allow one to perform MS/MS analysis on the multiply charged parent ions, localizing where the deuterium atoms are incorporated. Analysis of the MS/MS data is continuing but is complicated by the fact that various multiply charged product ions $(+1,+2$, and +3$)$ are present.

\section{Acknowledgments}

The authors acknowledge Alan Friedman and Teresa Kubiak of Upjohn for generously providing GRF analogs; David Wagner of Glaxo for technical assistance; and Dan Kassel, Larry Shampine, and Jian-Mei Ding of Glaxo for useful discussions.

\section{References}

1. Fenn, J. B.; Mann, M.; Meng, C. K.; Wong, S. F.; Whitehouse, C. M. Mass Spectrom. Rev. 1990, 9, 37-70.

2. Covey, T. R,; Bonner, R. F.; Shushan, B. I.; Henion, J. D. Rapid Commun. Mass Spectrom. 1988, 2, 249-256.

3. Chowdhury, S. K.; Katta, V.; Chait, B. T. I. Am. Chem. Soc 1990, 112, 9012-9013

4. Loo, J. A.; Edmonds, C. G.; Usdeth, H. R.; Smith, R. D. Anal. Chem. 1990, 62, 693-698.

5. Katta, V.; Chait, B. T. Rapid Commun. Mass Spectrom. 1991, 5, 214-217.

6. Le Blanc, J. C. Y.; Beuchemin, D.; Siu, K. W. M.; Guevremont, R.; Berman, S. S. Org. Mass Spectrom. 1991, 26, 831-839.

7. Loo, J. A.; Ogorzalek-Loo, R. R.; Usdeth, H. R.; Edmonds, C. G.; Smith, R. D. Rapid Commun. Mass Spectrom. 1991, 5, 101-105.

8. Guevremont, R.; Siu, K. W. M.; Le Blanc, J. C. Y.; Berman, S. S. I. Am. Soc. Mass Spectron. 1992, 3, 216-224.

9. Vance, M. L. Clin. Chem. 1990, 36, 415-420.

10. Clore, G. M.; Martin, S. R: Gronenborn, A. M. 1. Mol. Biol 1986, 91, 553-561.

11. Theriault, Y.; Boulanger, Y.; Saunders, J. K. Biopolymers 1988, $27,1897-1904$

12. Brunger, A. T.; Clore, G. M.; Gronenborn, A. M.; Karplus, M. Protein Eng. 1987, 5, 399-406.

13. Tou, J. S.; Kaempfe, L. A.; Vineyard, B. D.; Buonomo, F. C.; Della-Fera, M. A.; Baile, C. A. Biochem. Biophys. Res. Commun. $1986,139,763-770$. 
14. Kloosterman, D. A.; Scahill, T. A.; Hillman, R. M.; Cleary, D. L.; Kubiak, T. M. Peptide Res. 1991, 4, 72-78.

15. Stevenson, C. L.; Donlan, M. E.; Friedman, A. R; Borchardt, R. T. Int. I. Pept. Protein Res. 1993, in press.

16. Greenfield, N.; Fasman, G. D. Biochemistry 1969, 8, 4108-4116.

17. Chen, Y. H.; Yang, J. T.; Chat, K. H. Biochemistry 1974, 13, 3350-3359.

18. Chen, Y. H.; Yang, J. T. Biochem. Biophys. Res. Commun. 1971, $44,1285-1291$.

19. Chakrabartty, A.; Ananthrananarayanan, V. S; Hew, C. L. J. Biol. Chem. 1989, 264, 11307-11312.

20. Chou, P. Y.; Fasman, G. D. Biochemistry 1974, 13, 222-245.
21. Lyu, P. C.; Liff, M. I.; Marky, L. A.; Kallenbach, N. R. Science $1990,250,669-673$.

22. Felix, A. M.; Heimer, E. P.; Wang, C. T; Iambrns, T. J.; Fournier, A.; Mowles, T. F.; Maines, S.; Campbell, R. M.; Wegrzynski, B. B.; Toome, V; Fry, D.; Madison, V. S. Int. J. Pept. Protein Res. 1988, 32, 441-454.

23. Nelson, J. W.; Kallenbach, N. R. Biochemistry 1989, 28 , $5256-5261$.

24. Blundell, T.; Barlow, D.; Borkakot, N.; Thornton, J. Nature 1983, 306, 281- 283.

25. Mann, M.; Meng, C. K.; Fenn, J. B. Anal. Chem. 1989, 61, $1702-1708$. 